\title{
Immunohistochemical Profile and 47-Gene Next-Generation Sequencing (NGS) Solid Tumor Panel Analysis of a Series of 13 Strumal Carcinoids
}

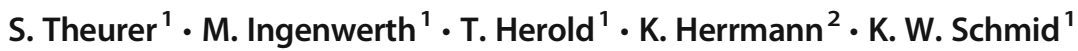

Published online: 2 March 2020

(C) The Author(s) 2020

\begin{abstract}
Strumal carcinoid is an extraordinary rare tumor of the ovary consisting of thyroid tissue intermixed with neuroendocrine tumor component. The cellular origin of strumal carcinoids has been an area of debate. There is also little data on detailed immunohistochemical and molecular characteristics of these neoplasms. For this reason, this series investigated the characteristics of a series of 13 strumal carcinoids using immunohistochemical markers and a 47-gene next-generation sequencing (NGS) solid tumor panel analysis. Both cellular components showed thyroglobulin expression in all tumors. TTF-1 expression was noted in both cellular components of 11 cases. Chromogranin A was positive in both components of most tumors $(n=12,92.3 \%$ in the neuroendocrine component and $n=10,76.9 \%$ in the thyroid follicular component). Synaptophysin stained the neuroendocrine component of all cases, and it was also identified in the follicular thyroid component of a single case. All tumors were negative for CDX2 and calcitonin. ISLET1 was positive in the neuroendocrine component of 8 cases $(6.5 \%)$. With the exception of one case, all tumors were positive for SSTR2a. The tumors were associated with a low Ki67 labeling index. All cases were microsatellite stable and no pathogenic mutations were identified using a 47-gene NGS solid tumor analysis. This series underscored that strumal carcinoids are distinct neuroendocrine tumors. The synchronous expression for thyroid follicular epithelial and neuroendocrine differentiation biomarkers may suggest a precursor cell origin displaying mixed-amphicrine differentiation. While strumal carcinoids can be diagnosed by their typical morphology and immunohistochemical profile, frequent SSTR expression may serve as a potential theranostic biomarker in the management of affected patients. In addition, the absence of common driver mutations in the NGS solid tumor panel may suggest that these neoplasms seem to be genetically unrelated to follicular epithelial-derived thyroid tumors and potentially different than other commonly identified well-differentiated neuroendocrine neoplasms. Therefore, further studies focusing on molecular characteristics of this entity are still needed.
\end{abstract}

Keywords Strumal carcinoid $\cdot$ Struma ovarii $\cdot$ Somatostatin receptor $\cdot$ SSTR $\cdot$ Neuroendocrine tumor

\section{Introduction}

Carcinoid tumors of the ovary are rare and account for less than $1 \%$ of all malignant neoplasm of the ovary [1]. They are subclassified into 4 types: insular, mucinous, trabecular, and strumal. Among these, strumal carcinoids are extremely rare and are composed of thyroid tissue admixed with

S. Theurer

Sarah.Theurer@uk-essen.de

1 Institute of Pathology, University Hospital Essen, University of Duisburg-Essen, Hufelandstr, 55, 45147 Essen, Germany

2 Institute of Nuclear Medicine, University Hospital Essen, University of Duisburg-Essen, Essen, Germany neuroendocrine tumor displaying insular or trabecular growth. These can reach a large tumor size and can be seen in association with elements of teratoma [2]. Several case reports identified that patients can manifest with severe obstipation due to the antiperistaltic effect of peptide YY, which seems to be frequently produced by tumor cells [3-5].

The cellular origin of strumal carcinoids has been an area of discussion since their first description in the 1970s. It seems to be proven that these are pure neuroendocrine tumors, as both neoplastic components, histologically presenting as "follicular thyroid" and "insular/trabecular neuroendocrine" components, contained neurosecretory granules $[6,7]$. In addition, an origin of C-cell was also postulated given immunohistochemical calcitonin expression in some cases [8]. The vast majority of strumal carcinoids are biologically indolent and curable by oophorectomy [1]. Only very few cases with 
metastatic disease were described $[9,10]$. Only one tumorassociated death has been published [11]. Due to the rarity of strumal carcinoids, knowledge about this tumor entity has been mostly generated from case reports $[1,12,13]$. In addition, a large series of 50 cases was published in 1980 addressed to the description of macroscopic and microscopic architectural features as well as the clinical outcome [11]. Expression of somatostatin receptors (SSTR) $2 \mathrm{a}$ and 5 is common in most well-differentiated neuroendocrine tumors [14] and these have been served as theranostic biomarkers [15]. Although SSTR expression was reported in a previous report of strumal carcinoid, the expression profile of SSTRs is not systematically investigated in these neoplasms. In addition, virtually nothing is known about molecular genetic alterations of strumal carcinoids. For instance, it is unknown if these tumors harbor similar alterations as seen in thyroid neoplasms and/or other neuroendocrine neoplasms. In light of the knowledge gap, this series investigated the characteristics of a series of 13 strumal carcinoids using immunohistochemical (bio)markers and a 47-gene next-generation sequencing (NGS) solid tumor panel analysis.

\section{Material and Methods}

We reviewed a case series of 13 strumal carcinoids, collected from surgery material in Germany between 2008 and 2019 and send to us for second opinion. Every case was seen and diagnosed by two specialists for thyroid and neuroendocrine pathology (KWS and ST). The (female) patients presented with a median age of 52.07 years (range 26-78 years). Tumor size varied from 0.3 to $4.2 \mathrm{~cm}$ (median size $1.35 \mathrm{~cm}$ ).

\section{Immunohistochemical Staining}

We performed immunohistochemical staining for thyroglobulin (DAKO, clone polyclonal rabbit, dilution 1:20000), TTF-1 (Roche, clone 8G7G3/1 mouse, RTU), chromogranin A (Leica, clone 5H7, dilution 1:100), synaptophysin SP11 (Cell Marque, clone MRQ-40 rabbit, RTU), Ki67 (Roche, clone 30-9, RTU), ISLET1 (Abcam, clone polyclonal rabbit, dilution 1:1000), CDX2 (Zytomed, clone EPR2764, dilution 1:500), calcitonin (DAKO, clone polyclonal rabbit, dilution 1:30000), SSTR2a (Zytomed, clone polyclonal rabbit, dilution 1:50), and SSTR5 (Zytomed, clone polyclonal rabbit, dilution 1:40). All stainings were performed on the Ventana Benchmark Ultra System.

\section{Next-Generation Sequencing}

Multiplex PCR and purification were performed with the GeneRead DNAseq Custom Panel V2, GeneRead DNAseq Panel PCR Kit V2 (QIAgen), and Agencourt ${ }^{\circledR}$ AMPure ${ }^{\circledR}$
XP Beads (Beckmann). A total amount of 45 ng DNA was used to perform multiplex PCR (four primer pools with $10 \mathrm{ng}$ / primer pool+ $10 \%$ excess volume). Library preparation was performed using NEBNext Ultra DNA Library Prep Set for Illumina (New England Biolabs; NEB), according to the manufacturer's recommendations. The pooled library was sequenced on MiSeq (Illumina; $2 \times 150$ bases paired-end run) and analyzed by the Biomedical Genomics Workbench (CLC Bio, QIAgen). Within the Biomedical Genomics Workbench, demultiplexed paired-end sequencing data was mapped to human genome (version hg19). A local realignment was performed to reach better alignment quality, especially for regions with small insertions or deletions. All reads which were mapped outside of targeted regions were deleted after the mapping process. In a filtering step, all reference variants and variants found in dbSNP common, 1000 genome project, and HapMap were deleted. An allele frequency of minimum $5 \%$ and coverage of at least 50 mapped reads were applied. For targeted sequencing, a customized panel was designed containing regions of interest. The customized panel included 47 genes. The analyzed genes and exons are listed in Table 1. The regions were covered by a total of 2193 amplicons and the

Table 1 Genes and exons included in NGS analysis

\begin{tabular}{|c|c|c|c|}
\hline Gene & Exons & Gene & Exons \\
\hline AKT1 & All & MAPK3 & All \\
\hline AKT2 & All & MDM2 & All \\
\hline ARID1A & All & MET & $3,8,11,14,19$ \\
\hline ARID1B & All & MLH1 & All \\
\hline ATM & All & MSH2 & All \\
\hline BAP1 & All & NF1 & All \\
\hline BCLAF1 & All & NRAS & 2.4 \\
\hline BRAF & 11.15 & PALB2 & All \\
\hline BRCA1 & All & PBRM1 & All \\
\hline BRCA2 & All & PDGFRa & $12,14,18$ \\
\hline EGFR & $18-21$ & PIK3CA & $3,5,10,16,21$ \\
\hline ERBB2 & $5,6,15,20,23,29$ & PTEN & All \\
\hline GNA11 & All & RAF1 & All \\
\hline GNAQ & All & RNF43 & All \\
\hline GNAS & All & RPA1 & All \\
\hline IDH1 & 4 & SF3B1 & $14,15,16$ \\
\hline IDH2 & 4 & SMAD4 & All \\
\hline KDM6A & All & SMARCA2 & All \\
\hline KIT & $9-11,13,17,18$ & SMARCA4 & All \\
\hline KRAS & 2.4 & SMARCB1 & All \\
\hline MAP2K1 & All & STK11 & All \\
\hline MAP2K2 & All & ТР53 & All \\
\hline \multirow[t]{2}{*}{ MAPK1 } & All & $\mathrm{TSC} 1$ & All \\
\hline & & $\mathrm{TSC} 2$ & All \\
\hline
\end{tabular}


total length of targeted regions was 176,370 . For all samples, an average coverage of approximately $2200 \times$ was obtained.

\section{Microsatellite Analysis}

DNA was isolated from FFPE Material and the Microsatellite Instability Analysis System (Promega) for Microsatellite Markers BAT-25, BAT-26, NR-21, NR-24, and MONO-27 was performed and analyzed with a 3500 Genetic Analyzer according to manufacturer's protocol (Applied Biosystems).

\section{Results}

\section{Histomorphology}

All tumors included in the series were composed of normalappearing "thyroid tissue" with follicular architecture admixed with varying amount of trabecular, insular, or nested areas resembling well-differentiated neuroendocrine tumors of other origins (Fig. 1). The nuclei of the neuroendocrine tumor component were round and enlarged with a characteristic pepper-and-salt chromatin pattern. No necrosis or mitotic activity was detected.

\section{Immunohistochemistry}

Immunohistochemical staining was evaluated for both components separately. Both cellular components showed thyroglobulin expression in all tumors. TTF-1 expression was noted in both cellular components of $11(84.6 \%)$ cases. Chromogranin A was also positive in both components of most tumors $(n=12,92.3 \%$ in the neuroendocrine component and $n=10,76.9 \%$ in the thyroid follicular component). Synaptophysin stained the neuroendocrine component of all cases, and it was also identified in the follicular thyroid component of a single case (7.7\%). Twelve cases had a Ki67 proliferation index of $<2 \%$. One case had a Ki67 proliferation index of 5\%. No mitotic figures are noted. All tumors were negative for CDX2 and calcitonin. Staining for ISLET1 showed positive nuclear immunostaining exclusively in the neuroendocrine component of 8 cases (6.5\%). SSTR2a was immunohistochemically detected in the neuroendocrine component of 12 out of 13 cases $(92.3 \%)$. Five cases were also immunoreactive for SSTR5 (41.7\%) in the neuroendocrine component. The "thyroid-like" tumor component was completely negative for both SSTRs in all cases. Only one case was negative for both SSTR2a and SSTR5. Details on staining results are listed in Table 2 and displayed in Fig. 2.

\section{DNA-Sequence Analyses of Microsatellites}

Sequence analyses of the microsatellites NR-21, BAT-26, BAT-25, NR-24, and MONO-27 revealed stable conditions in all cases.

\section{Next-Generation Sequencing}

Molecular genetic investigations via next-generation sequencing with 47 genes, 2193 amplicons, and a total length of targeted regions of 176,370 (see Table 1) did not detect any known or clinically relevant mutations.

\section{Discussion}

Strumal carcinoids are extraordinary rare tumors. Our morphological and clinical knowledge is mainly based on case reports, which were published over the last five decades in the literature. Since its first description in 1970, only one larger series of 50 cases analyzed architectural features of this disease. We hereby present the first large series of 13 cases of strumal carcinoids focusing on immunohistochemical biomarker expression related to thyroid follicular epithelial cells and neuroendocrine differentiation along with a 47-gene NGS solid tumor panel analysis.

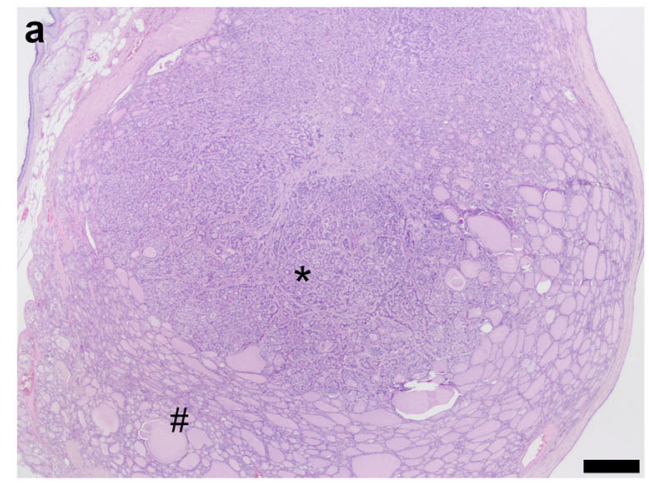

Fig. 1 Strumal carcinoid is composed of two distinct components. a Neoplastic thyroid-like tissue (\#) intermixed with tumor areas showing classical features of neuroendocrine tumors $(*), \mathrm{HE} \times 20$, scale bar

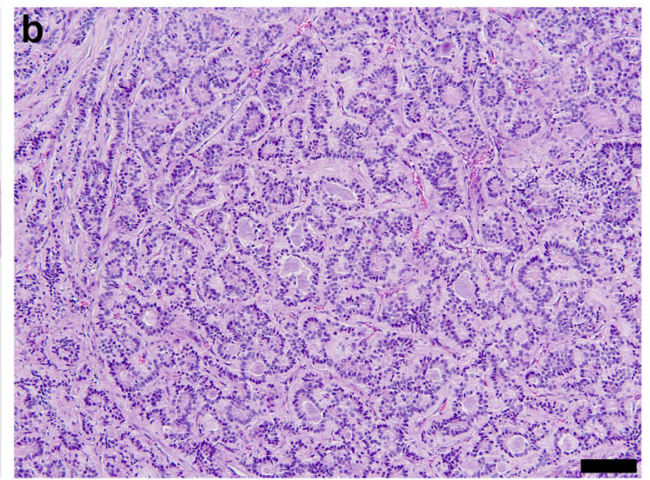

$500 \mu \mathrm{m}$. b Tumor cells of the neuroendocrine component show monomorphic round-shaped nuclei with trabecular- and rosette-like architecture, $\mathrm{HE} \times 100$, scale bar $100 \mu \mathrm{m}$ 
Table 2 Expression of different immunohistochemical markers, divided according to the two different tumor components of strumal carcinoid

\begin{tabular}{lll}
\hline Immunohistochemical marker & \multicolumn{2}{l}{ Positive staining result, $n(\%)$} \\
\cline { 2 - 3 } & Follicular component & Neuroendocrine component \\
\hline Chromogranin & $10(76.9)$ & $12(92.3)$ \\
Synaptophysin & $1(8.3)$ & $13(100)$ \\
Thyroglobulin & $13(100)$ & $13(100)$ \\
TTF1 & $11(84.6)$ & $10(84.6)$ \\
ISLET1 & $9(69.2)$ & $5(38.4)$ \\
CDX2 & 0 & 0 \\
Calcitonin & 0 & 0 \\
SSTR2a & 0 & $12(92.3)$ \\
SSTR5 & 0 & $6(46.1)$ \\
\hline
\end{tabular}

Histomorphological features indicated that these tumors consist of a "normal thyroid-like" component and a neuroendocrine component, which raised the question whether strumal carcinoids are distinct tumors themselves or two separate tumors in which the neuroendocrine tumor represents a somatic malignancy arising in the background of ovarian teratoma with thyroid follicular epithelial proliferation. There is

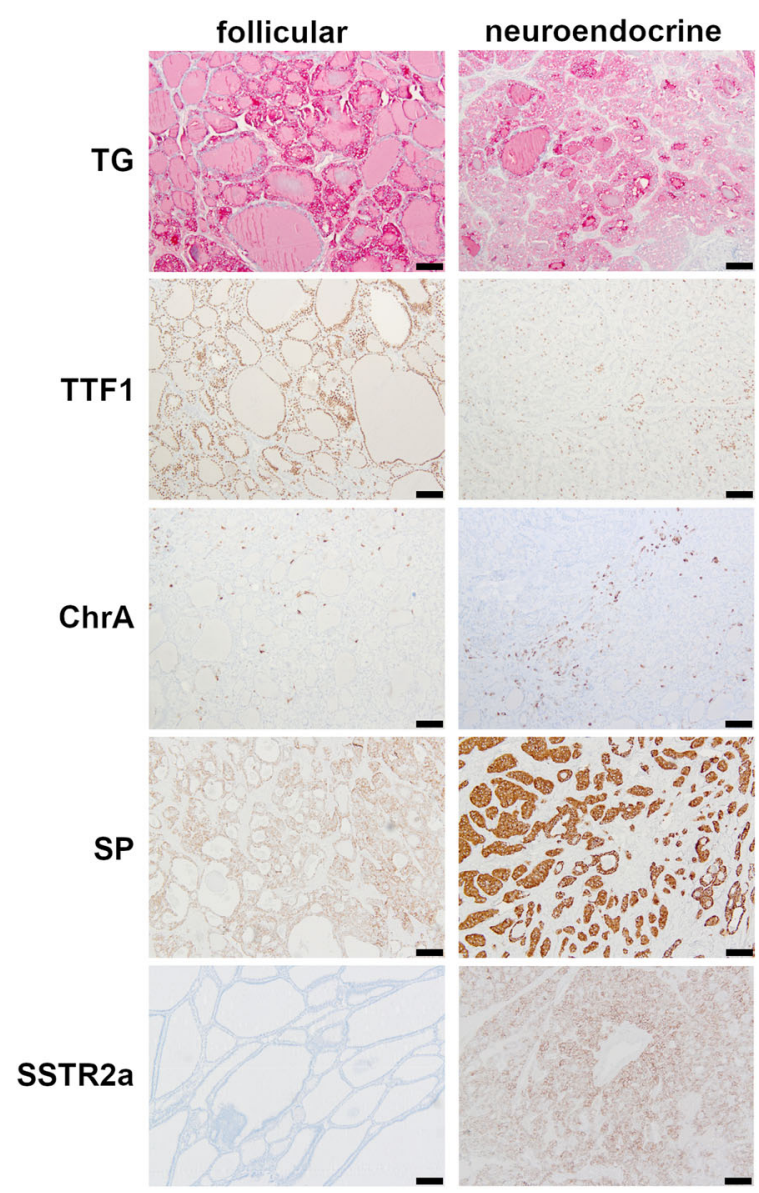

Fig. 2 Immunohistochemical staining for different antibodies in both components of strumal carcinoid: follicular tumor component on the left and tumor areas resembling neuroendocrine tumor on the right side no comparable type of primary tumor in the thyroid. Even the very rare mixed medullary and follicular carcinoma cannot be compared with this entity since the follicular epithelial component consists of either papillary thyroid carcinoma or follicular thyroid carcinoma [2].

Earlier investigations questioned the origin of strumal carcinoids. Interestingly, different studies came up with

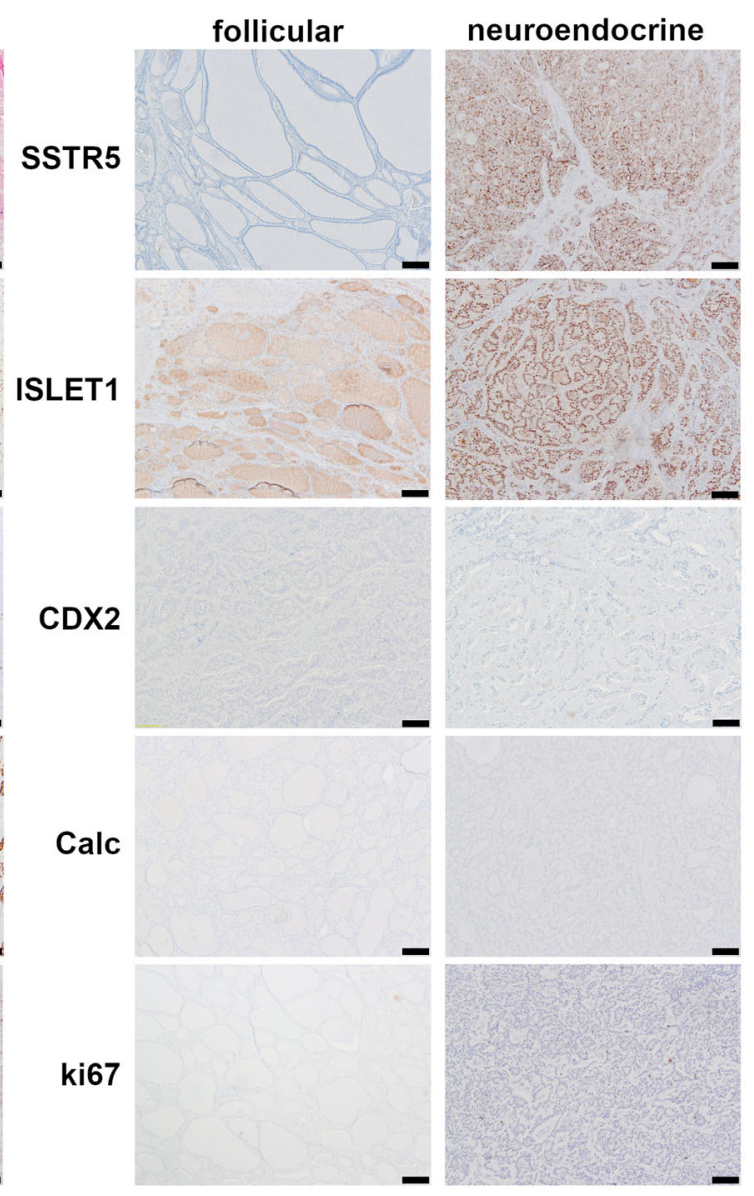

of the figure $(\mathrm{TG}=$ thyroglobulin, $\mathrm{ChrA}=$ chromogranin $\mathrm{A}, \mathrm{SP}=$ synaptophysin, Calc $=$ calcitonin). Magnification $\times 100$, scale bar $100 \mu \mathrm{m}$ 
different conclusions. In some ultrastructural studies, cytoplasmic neurosecretory granules suggested these neoplasms as a pure neuroendocrine tumor with follicular structures [6, 7]. Several subsequent studies argued against these considerations and showed thyroglobulin expression in both parts of the tumor or at least in transition between follicular and insular structures [16, 17]. Hamazaki et al. showed TTF-1 positivity only in follicular parts of the tumor in 2 cases, and concluded that the nested "neuroendocrine" part of the tumor has no thyroidal differentiation [18]. In this series, we also detected thyroid differentiation markers (thyroglobulin and TTF1) and neuroendocrine markers (chromogranin and synaptophysin) in both cellular components. These findings may support the theory of a tumor arising from a single precursor cell, harboring mixed-amphicrine features of both follicular epithelial cell of the thyroid (e.g., immunohistochemical features) and neuroendocrine cells (e.g., neurosecretory granules in electron microscopy, various neuroendocrine markers). One can hypothesize that the mixed morphology may occur during tumorigenesis represented by a thyroid-like follicular and a trabecular/ insular/solid neuroendocrine-like growth pattern. An origin of C-cells was also considered for these tumors based on calcitonin reactivity in some reported cases $[19,20]$. Our thorough immunohistochemical analysis failed to show any calcitonin expression in these neoplasms. Although commercially available calcitonin antibodies in the early years may have showed nonspecific results, we cannot exclude that in some cases a few cells may express calcitonin as seen in other neuroendocrine neoplasms. However, it seems to be highly unlikely that strumal carcinoids represent a tumor of C-cell origin or even a medullary thyroid carcinoma arising within a struma ovarii. These considerations can also be supported by the fact that C-cells do not express thyroglobulin.

In previous reports, peptide YY was detected (a peptide hormone produced by neuroendocrine cells of distal small intestine and most L-cells that are seen in the gastrointestinal tract and enriched in the colon) in strumal carcinoids. This has led to the assumption that the origin of strumal carcinoids may be of midgut- or hindgut-derived neuroendocrine precursor cells. The majority of neuroendocrine cells of the small intestine usually express CDX2 [21]. While this series did not assess peptide YY expression in these tumors, negative CDX2 expression in the current series may argue against the origin of midgut-derived neuroendocrine cells.

As described in former case reports [22], this series also confirmed a frequent expression rate of SSTRs (especially SSTR2a) in strumal carcinoids. Only one single case failed to show SSTR ( $2 \mathrm{a}$ and 5 ) expression. This case did not differ in its morphologic appearance, revealed no elevated Ki67 proliferative activity, and had a similar immunohistochemical profile compared with those with positive SSTRs.

Normal thyroid tissue is typically negative for SSTR2a; SSTR 5 can be very rarely detected in individual cells. The follicular component of strumal carcinoids lacked SSTR2a and SSTR5 expressions, which is in line with former investigations regarding expression of SSTRs in normal tissues [23].

Expression of somatostatin receptors is well known in differentiated neuroendocrine tumors of other origins and is used for diagnostic and therapeutic options [24]. While it remains unclear whether SSTRs are expressed in metastatic strumal carcinoids, there are good reasons from available data from other neuroendocrine neoplasms to assume that STTRs can be expressed in metastatic strumal carcinoids.

Interestingly, NGS analysis of 47 genes (with 2193 amplicons and a total length of targeted regions of $176,370)$ did not reveal any known pathogenic and/or clinically relevant mutations identified commonly in thyroid carcinoma and neuroendocrine tumors. While the existing solid tumor panel used could not identify possible driver mutations for development of strumal carcinoids, known driver mutations of neuroendocrine tumors (e.g., PTEN mutations in pancreatic neuroendocrine tumors or genes of the mTOR signaling pathway in neuroendocrine tumors of the small intestine and the lung [25]) were not detected in this series. Additionally, we could not detect the typical common driver mutations of differentiated thyroid carcinoma genes related to the MAP-Kinase signaling pathway [26], including $B R A F$ and $R A S$.

In the literature, malignant behavior of strumal carcinoids seems to be restricted to very few cases. In one malignant case, the primary tumor was found to be calcitonin-positive; however, calcitonin was not found in our series. Additionally, the metastatic tumor exhibited glandular/follicular structures, lacking any markers of neuroendocrine differentiation [9]. Therefore, one may question if the case described represents a true strumal carcinoid. A second report described a bilateral case of strumal carcinoid with metastases originating from the neuroendocrine component. CDX2 immunoreactivity was found both in the putative primary tumors and their metastases [10]. Since CDX2 is generally considered as a marker of intestinal neuroendocrine tumors (we were unable to immunelocalize CDX2 in the strumal carcinoids of our series), it also remains questionable whether this case constitutes a genuine metastatic strumal carcinoid or rather represents a case of metastatic (intestinal) neuroendocrine tumor in an ovarian teratoma with thyroid tissue. A third case report described a strumal carcinoid with metastatic spread to the contralateral ovary, the uterine corpus, and the lung. As the contralateral ovary showed similar histology, it was assumed to be metastatic. A potential bilateral occurrence of the disease should also be 
considered. The metastasis to the uterine corpus was confirmed by histology, showing only neuroendocrine differentiation. Immunohistochemical markers were also not performed both in the presumed primary tumor as well as in metastases. Additionally, the clinically identified lung metastasis was not histologically confirmed [27].

\section{Conclusion}

In summary, strumal carcinoids of the ovary are rare peculiar subtype of ovarian carcinoid tumors with unique features of both thyroid follicular cell and neuroendocrine differentiation, presumably deriving from one precursor cell. While these neoplasms can be diagnosed by their typical morphology and immunohistochemical profile, high frequency of SSTR2a expression may serve as a potential theranostic biomarker in the management of affected patients. While we acknowledge the limitations of NGS panel applied, the absence of common driver mutations in the NGS solid tumor panel may suggest that these neoplasms seem to be genetically unrelated to follicular epithelial-derived thyroid tumors and potentially different than other commonly identified welldifferentiated neuroendocrine neoplasms. Therefore, further studies focusing on molecular characteristics of this entity are still needed.

Author Contributions All authors contributed to the study conception and design. Material preparation, data collection, and analysis were performed by Sarah Theurer, Thomas Herold, and Marc Ingenwerth. The first draft of the manuscript was written by Sarah Theurer and all authors commented on previous versions of the manuscript. All authors read and approved the final manuscript.

Funding Information Open Access funding provided by Projekt DEAL.

\section{Compliance with Ethical Standards}

Conflict of Interest The authors declare that they have no conflict of interest.

Open Access This article is licensed under a Creative Commons Attribution 4.0 International License, which permits use, sharing, adaptation, distribution and reproduction in any medium or format, as long as you give appropriate credit to the original author(s) and the source, provide a link to the Creative Commons licence, and indicate if changes were made. The images or other third party material in this article are included in the article's Creative Commons licence, unless indicated otherwise in a credit line to the material. If material is not included in the article's Creative Commons licence and your intended use is not permitted by statutory regulation or exceeds the permitted use, you will need to obtain permission directly from the copyright holder. To view a copy of this licence, visit http://creativecommons.org/licenses/by/4.0/.

\section{References}

1. Antovska, V.S., Trajanova M., Krstevska I., Gosheva I., Chelebieva J., Prodanova I., Ovarian Strumal Carcinoid Tumour: Case Report. Open Access Maced J Med Sci, 2018. 6(3): p. 540-543.

2. Lloyd, R.V., et al., WHO Classification of Tumours of Endocrine Organs. 2017: International Agency for Research on Cancer.

3. Noh, H.K., Kwon B.S., Kim Y.H., Lee N.K., Choi K.U., Suh D.S., Lee D.H., Kim K.H., Peptide YY producing strumal carcinoid tumor of the ovary in a postmenopausal woman: a rare cause of chronic constipation. Obstet Gynecol Sci, 2017. 60(6): p. 602-607.

4. Matsuda, K., T. Maehama, and K. Kanazawa, Strumal carcinoid tumor of the ovary: a case exhibiting severe constipation associated with PYY. Gynecol Oncol, 2002. 87(1): p. 143-145.

5. Matsunami, K., et al., Peptide YY producing strumal carcinoid tumor of the ovary. Eur J Gynaecol Oncol, 2011. 32(2): p. 201-2.

6. Hart, W.R. and J.A. Regezi, Strumal carcinoid of the ovary: ultrastructural observations and long-term follow-up study. Am J Clin Pathol, 1978. 69(3): p. 356-359.

7. Livnat, E.J., Scommegna A., Recant W., Jao W., Ultrastructural observations of the so-called strumal carcinoid of the ovary. Arch Pathol Lab Med, 1977. 101(11): p. 585-589.

8. Dayal, Y., A.H. Tashjian, Jr., and H.J. Wolfe, Immunocytochemical localization of calcitonin-producing cells in a strumal carcinoid with amyloid stroma. Cancer, 1979. 43(4): p. 1331-1338.

9. Armes, J.E. and A.G. Ostor, A case of malignant strumal carcinoid. Gynecol Oncol, 1993. 51(3): p. 419-423.

10. Borghese, M., Razzore P., Ferrero A., Daniele L., Mariani L.L., Sgro L.G., de Rosa G., Biglia N., Metastatic Bilateral Strumal Carcinoid: A Case Report and Review of the Literature. Anticancer Res, 2019. 39(9): p. 5053-5056.

11. Robboy, S.J. and R.E. Scully, Strumal carcinoid of the ovary: an analysis of 50 cases of a distinctive tumor composed of thyroid tissue and carcinoid. Cancer, 1980. 46(9): p. 2019-2034.

12. Ciobanu Apostol, D.G., BuȚureanu T., Socolov D.G., Scripcaru D.C., Rosin O.L., Lozneanu L., Ovarian strumal carcinoid - case report. Rom J Morphol Embryol, 2017. 58(3): p. 1035-1040.

13. Lenicek, T., Tomas D., Soljacić-Vranes H., Kraljević Z., Klarić P., Kos M., Kos M., Strumal carcinoid of the ovary: report of two cases. Acta Clin Croat, 2012. 51(4): p. 649-653.

14. Mai, R., Kaemmerer D., Träger T., Neubauer E., Sänger J., Baum R.P., Schulz S., Lupp A., Different somatostatin and CXCR4 chemokine receptor expression in gastroenteropancreatic neuroendocrine neoplasms depending on their origin. Sci Rep, 2019. 9(1): p. 4339.

15. Fani, M., G.P. Nicolas, and D. Wild, Somatostatin Receptor Antagonists for Imaging and Therapy. J Nucl Med, 2017. 58(Suppl 2): p. 61S-66S.

16. Greco, M.A., LiVolsi V., Pertschuk L.P., Bigelow B., Strumal carcinoid of the ovary: an analysis of its components. Cancer, 1979. 43(4): p. 1380-1388.

17. Senterman, M.K., Cassidy P.N., Fenoglio C.M., Ferenczy A., Histology, ultrastructure, and immunohistochemistry of strumal carcinoid: a case report. Int J Gynecol Pathol, 1984. 3(2): p. 232-240.

18. Hamazaki, S., Okino T., Tsukayama C., Okada S., Expression of thyroid transcription factor-1 in strumal carcinoid and struma ovarii: an immunohistochemical study. Pathol Int, 2002. 52(7): p. 458-462.

19. Blaustein, A., Calcitonin secreting struma-carcinoid tumor of the ovary. Hum Pathol, 1979. 10(2): p. 222-228. 
20. Ulbright, T.M., L.M. Roth, and C.E. Ehrlich, Ovarian strumal carcinoid. An immunocytochemical and ultrastructural study of two cases. Am J Clin Pathol, 1982. 77(5): p. 622-631.

21. Yang, Z., Klimstra D.S., Hruban R.H., Tang L.H., Immunohistochemical Characterization of the Origins of Metastatic Well-differentiated Neuroendocrine Tumors to the Liver. Am J Surg Pathol, 2017. 41(7): p. 915-922.

22. Kurabayashi, T., Minamikawa T., Nishijima S., Tsuneki I., Tamura M., Yanase T., Hashidate H., Shibuya H., Motoyama T., Primary strumal carcinoid tumor of the ovary with multiple bone and breast metastases. J Obstet Gynaecol Res, 2010. 36(3): p. 567-571.

23. Unger, N., Ueberberg B., Schulz S., Saeger W., Mann K., Petersenn S., Differential expression of somatostatin receptor subtype 1-5 proteins in numerous human normal tissues. Exp Clin Endocrinol Diabetes, 2012. 120(8): p. 482-489.

24. Pavel, M., O’Toole D., Costa F., Capdevila J., Gross D., Kianmanesh R., Krenning E., Knigge U., Salazar R., Pape U.F., Öberg K., Vienna Consensus Conference participants., ENETS
Consensus Guidelines Update for the Management of Distant Metastatic Disease of Intestinal, Pancreatic, Bronchial Neuroendocrine Neoplasms (NEN) and NEN of Unknown Primary Site. Neuroendocrinology, 2016. 103(2): p. 172-185.

25. Di Domenico, A., et al., Genetic and epigenetic drivers of neuroendocrine tumours (NET). Endocr Relat Cancer, 2017. 24(9): p. R315-R334.

26. Cancer Genome Atlas Research, N., Integrated genomic characterization of papillary thyroid carcinoma. Cell, 2014. 159(3): p. 676690.

27. Khadilkar, U.N., Pai R.R., Lahiri R., Kumar P., Ovarian strumal carcinoid-report of a case that metastasized. Indian J Pathol Microbiol, 2000. 43(4): p. 459-461.

Publisher's Note Springer Nature remains neutral with regard to jurisdictional claims in published maps and institutional affiliations. 